\title{
ANÁLISE BIO-BIBLIOMÉTRICA O CASO CESAR GOMES VICTORA
}

\author{
Juliana Gonçalves Reis \\ Universidade Federal Fluminense, Programa de Pós-graduação Saúde Materno-Infantil \\ julianareis20@gmail.com
}

Haydée Maria Correia da Silveira Batista Universidade Federal Fluminense, Programa de Pós-graduação Sistemas de Gestão haydeesilveira@id.uff.br

Adauto Dutra Moraes Barbosa

Universidade Federal Fluminense, Programa de Pós-graduação Saúde Materno-Infantil adutra@vm.uff.br

\author{
Martius Vicente Rodriguez y Rodriguez \\ Universidade Federal Fluminense, Departamento de Administração \\ martiusyrodriguez@gmail.com
}

\begin{abstract}
Resumo
Este estudo bio-bibliométrico tem como objetivo mapear a evolução da rede de colaboração e o acúmulo do capital científico do retratado - Cesar Gomes Victora ao longo de 40 anos. Optou-se pela base de dados bibliográficos Scopus como fonte principal para composição do corpus das redes de autores composto $(n=502)$ artigos de periódicos, separados em quatro coleções por décadas 1977-1986;1987-1996;1997-2006 e 2007-2016 para configuração das redes de colaboração e do acúmulo de capital científico em uma linha do tempo. Os resultados mostraram o crescimento expressivo do número de publicações e de colaborações nas quatro décadas. O número e a força de ligação ocorreram com maior expressão nos últimos 20 anos. A década mais produtiva foi de 2007 a 2016. O acúmulo do capital científico tanto institucional, como específico foram construídos em contextos local e global conferiram ao Cesar Gomes Victora autoridade internacional no campo materno-infantil.
\end{abstract}

Palavras-chave: Bio-bibliometria. Colaboração científica. Capital científico. Estudo de caso. Saúde maternoinfantil.

\section{BIO-BIBLIOMETRIC ANALYSIS THE CASE CESAR GOMES VICTORA}

\begin{abstract}
This bio-bibliometric study aims to map the evolution of the collaboration network and the accumulation of scientific capital of Cesar Gomes Victora over 40 years. We chose the Scopus bibliographic database as the main source for composing the authors' corpus $(\mathrm{n}=502)$ journal articles, separated into four collections by decades as 1977-1986, 1987-1996, 1997-2006, and 2007- 2016 for the configuration of collaboration networks and the accumulation of scientific capital in a timeline. The results showed the significant evolution in number of publications and collaborations during the four decades. The number and bonding strength have occurred most significantly over the past 20 years. The most productive decade was from 2007 to 2016 . The accumulation of both institutional and specific scientific capital were built in local and global contexts and gave Cesar Gomes Victora an international authority on the maternal and child field.
\end{abstract}

Keywords: Bio-bibliometric. Cientific colaboration. Scientific capital.

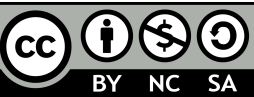

P2P \& INOVAÇÃO, Rio de Janeiro, v. 5 n. 2, p.133-153, Mar./Ago. 2019. 


\section{INTRODUÇÃO}

Os estudos sobre colaboração científica têm despertado interesse na gestão do conhecimento por evidenciar as relações entre atores de um ou mais campo de conhecimento. As redes de autores e coautores; instituiçõos e países; ordem de autores e tipo de contribuição e áreas de conhecimento entre outros elementos bibliográficos são aspectos explorados nesses métodos de investigação. O estudo de coautoria é uma das formas mais tangíveis e bem documentadas de colaboração científica, quase todos os aspectos das redes de colaboração científica podem ser rastreados de forma confiável, analisando as redes de coautoria por meio de métodos bibliométricos (GLANZEL E SCHUBERT ,2004, p.274).

Neste sentido, a análise bio-bibliométrica apresenta-se um método de mapear a colaboração científica de uma autoridade do campo de conhecimento e relacionar com os conceitos propostos pelo sociólogo francês Pierre Bourdieu, a qual define:

\footnotetext{
O campo científico enquanto sistema de relações objetivas entre posições adquiridas (em lutas anteriores), é o lugar, o espaço de jogo de uma luta concorrencial. O que está em jogo especificamente nessa luta é o monopólio da autoridade científica definida, de maneira inseparável, como capacidade técnica e poder social; ou, se quisermos, o monopólio da competência científica, compreendida enquanto capacidade de falar e de agir legitimamente (isto é, de maneira autorizada e com autoridade), que é socialmente outorgada a um agente determinado (BOURDIEU, 1983, pg 122 e 123).
}

Em sua outra obra, Os usos sociais da ciência, por uma sociologia clínica do campo científico, Bourdieu (2004) destaca que os campos são lugares de duas formas de poder que correspondem a duas espécies de capital científico: 1) Capital institucional ou social, está ligado a ocupação de posições importantes nas instituições científicas; 2) Capital específico, está ligado ao capital simbólico de atos de conhecimento e de reconhecimento, quase que exclusivamente do conjunto de seus pares. As duas espécies de capital científico levam tempo para acumular e têm um trabalho lento de colaboração.

O presente estudo propõe a metodologia da bio-bibliometria como abordagem da análise de redes de colaboração científica e do mapeamento de acúmulo do capital científico em ordem cronológica.

O campo científico da saúde materno-infantil apresenta complexidade e amplitude (multidisciplinaridade) que colaboram para a dispersão da informação científica, ocasionando limitações no mapeamento em sua totalidade, ao contrário de áreas mais delineadas, como pediatria, obstetrícia, nutrição e outras.

A saúde materno-infantil não é bem delimitada, pois inclui as áreas acima, como a saúde reprodutiva/anticoncepção, neonatologia, serviços de saúde, saúde pública, epidemiologia entre 
outras. Análises sobre a evolução da colaboração científica no nível micro, em relação à produção científica gerada na pesquisa em um campo específico, pode revelar o crescimento, a especificidade e amplitude de uma comunidade de pesquisa, como descreveram GonzalezAlcaide et.al. (2015) sobre a evolução dos padrões de colaboração na pesquisa em Psoríase.

Na necessidade de conhecer o desenvolvimento do campo da saúde materno-infantil, com foco para a saúde global e translação do conhecimento, optou-se por investigá-lo a partir da evolução da rede de colaboração e acúmulo do capital científico de uma autoridade com intensa sociabilidade científica, mobilidade e destaque internacional - Cesar Gomes Victora, médico, epidemiologista brasileiro considerado uma liderança mundial em saúde maternoinfantil (BOSELEY,2007).

Victora trabalha há quarenta anos no campo da saúde materno-infantil com contribuições imprescindíveis para políticas globais e nacionais. Atualmente, coordena o Centro Internacional de Equidade em Saúde, vinculado à Universidade Federal de Pelotas, cuja missão é monitorar a equidade em saúde e nutrição materno-infantil em todo o mundo, especialmente em países de baixa e média renda.

Este artigo tem como objetivo mapear a evolução da rede de colaboração e o acúmulo do capital científico do retratado - Cesar Gomes Victora ao longo de 40 anos.

\section{METODOLOGIA}

\subsection{TIPO DE ESTUDO}

Este estudo faz uma análise bio-bibliométrica. Segundo Hayashi (2014) “a bibliometria e a cienciometria fornecem um instrumental para mapear e extrair informações úteis para compreender a estrutura social e intelectual de campos científicos e os aspectos sociais, econômicos e políticos envolvidos na atividade científica”. Esta mesma autora, descreve os "retratos bio-bibliométricos", como uma modalidade de abordagem que permite ultrapassar o mero levantamento de dados bibliográficos correlacionando análise bibliométrica com a trajetória intelectual, acadêmica e científica do retratado (correspondência pessoal).

\subsection{FONTES DE DADOS}

Optou-se pela base de dados bibliográficos Scopus como fonte principal para composição do corpus das redes de autores, por ter cobertura internacional e multidisciplinar. 
Ressalta-se que outras fontes, como o currículo do retratado na Plataforma Lattes, as entrevistas concedidas a veículos de comunicação e as conferências ministradas disponíveis na internet foram consultadas para delineamento da trajetória biográfica e recorte temporal das décadas, conforme a figura 1 .

\subsection{COLETA DE DADOS}

A estratégia de busca foi construída a partir do número do Open Researcher and Contributor ID - ORCID do autor "0000-0002-2465-2180" sem limite de tempo, para identificar todos os registros bibliográficos. ORCID é um código alfanumérico digital para identificação do autor. Essa ferramenta tem como finalidade melhorar a capacidade de recuperação da informação, minimizar as perdas e a dispersão ocasionadas pela diversidade de grafia do nome do autor. No caso desta pesquisa, as variações de grafia do nome foram: Victora, Cesar Gomes; Victora, Cesar G.; Victora, C. G. e Victora, Cesar. Este código, proporciona integração com outras bases de dados bibliográficos, plataformas de submissão de artigos, agências de financiamento e repositórios institucionais entre outros. A data da coleta foi 10 de janeiro de 2017.

\subsection{CRITÉRIOS DE ELEGIBILIDADE}

Foram incluídos artigos científicos e editoriais com autoria ou coautoria de Cesar Gomes Victora até 2016 e excluídos artigos publicados em 2017, duplicatas, livros, capítulos de livros, resumos de eventos científicos e erratas.

\subsection{PROCESSO DE SELEÇÃO}

Foram identificados $(\mathrm{N}=575)$ documentos, excluídos $(\mathrm{n}=73)$ por não atenderem os critérios de inclusão e assim sendo, foi configurada a composição do corpus das redes de colaboradores em $(n=502)$ documentos.

\subsection{ORGANIZAÇÃO E ANÁLISE DOS DADOS}

Foram criadas quatro coleções organizadas em série histórica, a saber: C1 (1977-1986); C2 (1987-1996); C3 (1997-2006) e C4 (2007-2016) dos registros bibliográficos no gerenciador 
de referências Zotero, um software de acesso livre utilizado como ferramenta de apoio à pesquisa. A limpeza e padronização de nomes dos autores foi realizada para melhorar a precisão e qualidade dos dados. Para este estudo, considerou-se os nomes de autores recuperados pelo gerenciador de referências, independente da ordem e da declaração de contribuição de autoria.

Para caracterizar a evolução das redes de colaboração, as análises foram realizadas em três etapas. Na primeira etapa, as coleções depositadas no gerenciador de referências Zotero foram exportadas e importadas em formato RIS para processamento da visualização e análise das redes de autores no software livre VOSviewer Visualizing scientific landscapes do Center for Science and Technology Studies (CWST) da Universidade de Leiden, na Holanda. Na segunda etapa, foram calculadas para compor a série histórica as distribuições das frequências do número de documentos, número de autores, número de ligações e número da força da ligação. Os padrões de colaboração observados foram: Número de nós: número de autores que compõem a rede; número de links: número de relações entre os autores com ligações únicas ou repetidas e força de ligação: número da força de laços entre os autores.

$\mathrm{Na}$ terceira etapa, foi construído um quadro de síntese com a linha do tempo a partir de elementos do acúmulo do capital científico da trajetória de Cesar Victora relacionados com as estratégias, políticas e influências para a saúde materno-infantil.

Figura 1 - Fluxo do desenho do estudo

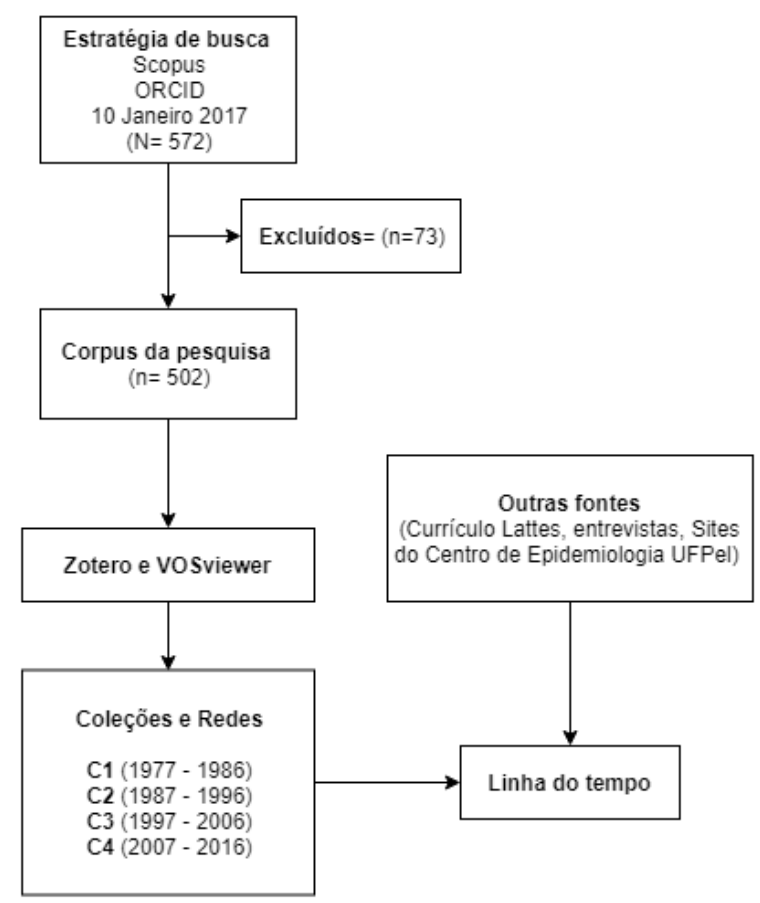




\section{RESULTADOS E DISCUSSÃO}

O gráfico 1 e a tabela 1 apresentam a série histórica da evolução das redes de colaboração científica de Cesar Victora. Sequencialmente, serão apresentadas quatro figuras de redes de colaboração em ordem cronológica. A seguir, o quadro 1 de síntese com a linha do tempo com o acúmulo de capital científico, as políticas de saúde materno-infantil e as influências.

Gráfico 1 - Série histórica da evolução da rede de colaboração científica. 1977-2016

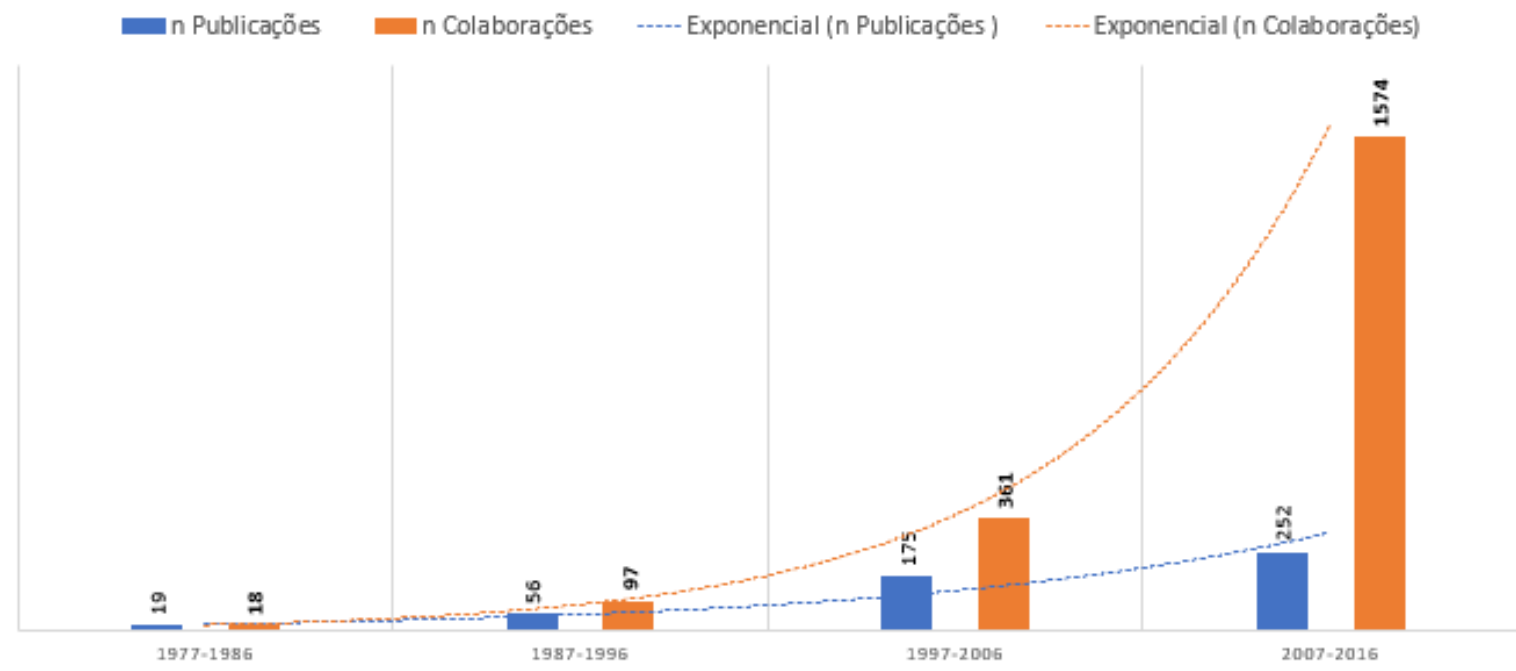

Fonte de dados: elaboração própria com dados da Scopus

Essas ilustrações mostram que houve crescimento exponencial nas quatro décadas. Os números de publicações e de colaborações elevaram consequentemente, o número de ligações e a força de ligação com maior expressão nos últimos 20 anos. A década mais produtiva, foi de 2007 a 2016.

Tabela 1 - Série histórica da evolução da rede de colaboração científica. 1977-2016.

\begin{tabular}{lcccc}
\hline \multirow{2}{*}{ Variáveis } & \multicolumn{4}{c}{ Série histórica } \\
\cline { 2 - 5 } & $\mathbf{1 9 7 7 - 1 9 8 6}$ & $\mathbf{1 9 8 7 - 1 9 9 6}$ & $\mathbf{1 9 9 7 - 2 0 0 6}$ & $\mathbf{2 0 0 7 - 2 0 1 6}$ \\
\cline { 2 - 5 } & $\mathrm{n}$ & $\mathrm{n}$ & $\mathrm{n}$ & $\mathrm{n}$ \\
\hline Publicações & 19 & 56 & 175 & 252 \\
\hline Autores & 18 & 97 & 361 & 1.574 \\
\hline Ligações & 42 & 576 & 3.826 & 334.344 \\
\hline Força de ligação & 91 & 997 & 5.680 & 375.769 \\
\hline
\end{tabular}

Fonte de dados: elaboração própria com dados da Scopus 
3.1 A PRIMEIRA DÉCADA, 1977 A 1986 - A FORMAÇÃO DA TRÍADE E A PRIMEIRA COORTE DE NASCIMENTO DE PELOTAS

Logo após a conclusão da graduação em Medicina, na Universidade Federal do Rio Grande do Sul (UFRGS) em 1976, Cesar Victora cursa Residência em Saúde comunitária, na mesma instituição. Nesse período, Kurt Kloetzel (1923-2007) saiu da Universidade de São Paulo (USP) e peregrinou por várias universidades em decorrência da perseguição política. Kurt foi discípulo de Samuel Pessoa (1898-1976), um dos precursores da medicina social no Brasil e ambos foram perseguidos pela ditadura militar. Essas universidades recém-criadas por onde ele passou foram Jundiaí, Mogi das Cruzes, Londrina e Pelotas, onde ele permaneceu por mais tempo e fundou o Departamento de Medicina Social (KLOETZEL, 2017).

Para constituir o quadro de professores do departamento, Kurt foi até o curso de Saúde Comunitária e convidou Cesar Victora e Nelson Blank para compor o recém-criado Departamento de Medicina Social da Universidade Federal de Pelotas, na época chamada de Leiga.

Na figura 2, cada ponto colorido ou nó representa um autor que interagiu em colaboração com Cesar Victora. O tamanho do nó reflete ao número de colaborações. A espessura da linha de conexão representa a força de ligação.

Observa-se as duas principais ligações, Fernando Barros e John Patrick Vaughan, formando uma tríade com Cesar Victora. Essa tríade na produção científica foi estabelecida no período do doutorado em epidemiologia, realizado entre 1980 e 1983, na London School of Hygiene and Tropical Medicine (LSHTM), da Universidade de Londres, na Inglaterra, na qual ambos foram orientados por John Patrick Vaughan e fortalecida nos estudos posteriores ao longo dos anos.

Fernando Celso Lopes Fernandes Barros, médico pediatra, professor na Universidade Católica de Pelotas, cursou mestrado em materno-infantil na Universidade de Londres e doutorado na LSHTM. Victora descreve como chegou até Vaughan "Alguns anos antes um professor inglês, David Morley, que orientou o Fernando no mestrado, nos apresentou o epidemiologista John Patrick Vaughan, nosso orientador no doutorado” (ZORZETTO, 2013). 
Figura 2 - Infográfico da rede de colaboração de Victora, CV.1977-1986 (Nós: autores que compõem a rede. Links: número de relações entre os autores com ligações únicas ou repetidas).

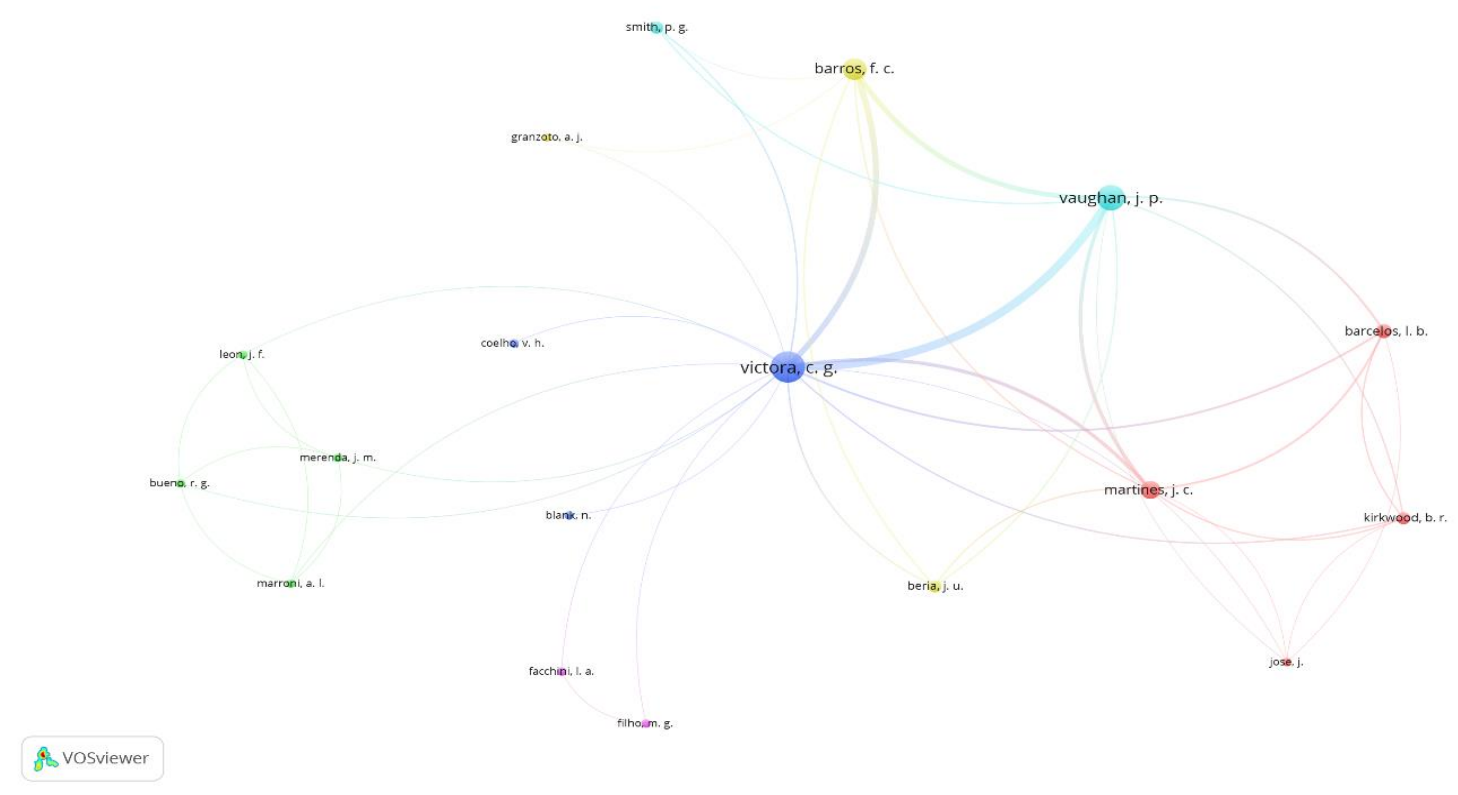

Fonte de dados: elaboração própria com dados da Scopus

Na tese de doutorado de Victora intitulada, Epidemiologia da saúde infantil no Rio Grande do Sul, Brasil. As inter-relações entre mortalidade, desnutrição, assistência médica e desenvolvimento agrícola. Esta pesquisa comparou a frequência de desnutrição no estado do Rio Grande do Sul. "Mostrei que no Sul, onde estavam os latifúndios, a mortalidade infantil era muito alta. Já na região norte, onde predominava o pequeno agricultor, que tinha sua terra, cultivava seus alimentos, a desnutrição e a mortalidade eram baixas." (ZORZETTO, 2013).

A linha de pesquisa, revela os efeitos do Relatório Lalonde, Uma Nova Perspectiva na Saúde dos Canadenses de 1974, a qual insere o conceito "campo da saúde" e propõe quatro elementos gerais: a biologia, os serviços de saúde, o meio ambiente e os estilos de vida (CANADA, 1974). Como sugere a fala de Victora:

Comecei a estudar mortalidade infantil e desnutrição. Sempre trabalhei com populações muito pobres, de favelas de Pelotas e Porto Alegre, e havia percebido que as crianças sempre voltavam [ao posto de saúde] com os mesmos problemas: diarreia, desnutrição, pneumonia. Eu tratava uma criança e ela voltava duas semanas depois com a mesma coisa. Elas viviam num ambiente de muita pobreza, não tinham água encanada, não eram amamentadas e estavam expostas a contaminações ambientais. Foi aí que resolvi ir para a epidemiologia e tentar fazer algo voltado para a prevenção das doenças. (ZORZETTO, 2013). 
Vale ressaltar que na época, a duração do aleitamento materno era inferior a três meses, o estímulo à oferta de líquidos aos recém-nascidos e lactentes, como chás, leite artificial e de vaca eram muito comuns.

Em 1983, Victora retorna ao Brasil, segue o acompanhamento da coorte de 1982 e avança os estudos no campo da saúde materno-infantil. Os primeiros resultados da Coorte de 1982 divulgados no Brasil, na Revista de Saúde Pública, em dois artigos, o primeiro artigo em 1984: Saúde perinatal em Pelotas, RS, Brasil: fatores sociais e biológicos ressalta o interesse por estudos epidemiológicos no período perinatal, assim como a importância de dois conjuntos de determinantes: 1- adequada estrutura socioeconômica e 2- eficiente sistema de saúde (BARROS et al. 1985). E o segundo artigo, em 1985: Estudo longitudinal das crianças nascidas em 1982 em Pelotas, RS, Brasil. Metodologia e resultados preliminares mostraram as potencialidades da utilização de dados na investigação dos determinantes pré e pós-natais da saúde infantil, assim como no estudo da interação entre fatores sociais e biológicos. Ainda destacam que "diversos indicadores de utilização de serviços de saúde foram também colhidos, o que permitirá seu uso na avaliação da cobertura e possivelmente da efetividade destes serviços" (VICTORA et al 1985).

O movimento para a construção de um sistema de saúde mais amplo não foi exclusivo dos eixos Rio de Janeiro, São Paulo e Bahia. Nessa década, havia engajamento em outras regiões do país, inclusive em Pelotas, como sugere o relato de Barros et al "a divulgação e debate destes estudos certamente indicarão quais as mudanças que devem ser efetuadas em nosso sistema atual. Esperamos que o Projeto Perinatal de Pelotas possa colaborar neste sentido" (BARROS et al. 1985).

A tríade formada por Cesar, Fernando e Patrick impulsionou o estudo da coorte de nascimentos de 1982, segundo o site do Centro de Pesquisas Epidemiológicas da UFPel:

\footnotetext{
O material deste estudo foi também utilizado para a tese de Doutorado em Epidemiologia do Professor Barros, na London School of Hygiene and Tropical Medicine da Universidade de Londres. Seu orientador de doutorado foi o Professor J. Patrick Vaughan, e o apoio financeiro ao projeto foi obtido de uma instituição de fomento à pesquisa do Canadá (IDRC). Ao final do estudo perinatal, juntou-se ao projeto o médico e professor da Universidade Federal de Pelotas (UFPel), Cesar Victora, que estava finalizando seu Doutorado, também na Universidade de Londres. Juntos, Barros e Victora preparam o primeiro acompanhamento da coorte, e todas as suas etapas subsequentes, até os dias de hoje (EPIDEMIOLOGIA UFPel 2017).
}

Esse primeiro grande estudo epidemiológico do Brasil, teve como objetivo inicial avaliar a influência que os fatores inerentes ao período do nascimento da criança mantinham em relação a sua saúde na infância. Esses fatores incluíram condições da saúde materna e da criança, peso ao nascer, alimentação e as condições ambientais entre outros. A pesquisa superou 
as expectativas, foi muito bem aceita e a OMS concedeu o financiamento para continuar o acompanhamento de cerca de seis mil pessoas. (EPIDEMIOLOGIA UFPel, 2017).

Destacamos na figura 2 as ligações periféricas representadas por colaborações iniciais na UFPel e uma ligação ponte, representada por José Carlos Martines, que faz conexão com outras ligações periféricas representadas pelo artigo Risk factors for malnutrition in Brazilian children: the role of social and environmental variables, em língua portuguesa, Fatores de risco para a desnutrição em crianças brasileiras: o papel das variáveis sociais e ambientais, publicado no Bulletin of the World Health Organization, em 1986. Martines é médico pediatra, com mestrado pela LSHTM e doutorado pela Universidade de Londres ambos em nutrição humana. Coordenou por mais de quinze anos o setor de pesquisa do Programa global de saúde da criança da OMS em Genebra.

Victora et al (1986) relataram os efeitos de indicadores socioeconômicos e ambientais sobre o estado nutricional de crianças em áreas urbanas e rurais do sul do Brasil. Das variáveis sociais estudadas, a renda familiar e o nível de educação do pai foram os dois fatores de risco que apresentaram as mais fortes associações com o estado nutricional. O nível de escolaridade da mãe, o status de ocupação do chefe da família, o número de irmãos e as origens étnicas da família também mostraram algum grau de associação, mas estes foram menos significativos quando a renda familiar foi incluída na análise. As variáveis ambientais, particularmente o tipo de habitação, o grau de aglomeração e o tipo de disposição de esgoto, também foram fortemente associados à desnutrição. Este tipo de análise multivariável, com controle estatístico através de métodos de regressão, era muito inovador naquela época, tanto no Brasil quanto em outros lugares, particularmente na área de saúde materno-infantil.

Esses achados são anteriores a criação do Sistema Único de Saúde do Brasil (SUS), em um momento histórico que os determinantes sociais de saúde ainda estavam buscando consolidação como objeto de investigação no campo da saúde materno-infantil e a Atenção Primária da Saúde (APS) ainda não era uma realidade estabelecida no país. Em um documento técnico do Ministério da Saúde, Assistência integral à saúde da criança, de 1984, marca uma linha política com ações básicas aos agravos mais frequentes e a mortalidade infantil com destaque para as principais diretrizes o aleitamento materno, orientação alimentar para o desmame, a imunização, o acompanhamento do crescimento e desenvolvimento (MINISTÉRIO DA SAÚDE, 1984). 
3.2 A SEGUNDA DÉCADA, 1987 A 1996, A FORMAÇÃO DE UMA COMUNIDADE EPISTÊMICA EM EPIDEMIOLOGIA E SAÚDE MATERNO-INFANTIL E A SEGUNDA COORTE DE NASCIMENTOS DE PELOTAS

A divulgação dos resultados preliminares da primeira coorte de nascimentos de Pelotas, já apresentavam contribuições para o campo da saúde materno-infantil. Entretanto, a segunda década tem como pontos altos a expansão de estudos de mapeamento da desnutrição e mortalidade infantil no Nordeste do Brasil em parceria com a Unicef, novas descobertas sobre amamentação e a criação em 1991 do Centro de Pesquisas Epidemiológicas (CPE) com o curso de pós-graduação em Epidemiologia como destaca Victora:

\begin{abstract}
Entre 1987 e 2001, fizemos inquéritos em nove estados. Havia índices altíssimos de mortalidade infantil [as taxas eram superiores a 100 mortes para cada mil nascimentos]. Interagimos com secretários de Saúde e conseguimos direcionar as linhas de atuação dos estados com base nos resultados da pesquisa. Mas nosso grupo era pequeno. Fernando e eu treinávamos de forma amadora os colegas interessados. Em 1990 vimos que precisávamos de gente que quisesse fazer epidemiologia. Então criamos o mestrado em 1991. Foi nossa maior sacada. (ZORZETTO, 2013).
\end{abstract}

Em 1993, início da segunda coorte de nascimentos de Pelotas, com a finalidade de comparar as características da saúde materno-infantil e evolução dos indicadores de saúde (Epidemiologia UFPel, 2017).

Nesse período, o Brasil avança com a construção do SUS, a implementação do Programa Saúde da Família em 1994 e a inserção do novo ator na Atenção Primária de Saúde, o Agente Comunitário de Saúde (ACS).

Na figura 3, observa-se o crescimento e o fortalecimento da rede de colaboração. É evidenciada a tríade mais fortalecida: Victora, Barros e Vaughan. Nessa rede, destaca-se três principais grupos de colaboração com produção científica relevante para a saúde maternoinfantil em descobertas e advocacia para políticas e translação do conhecimento.

No grupo verde representado pelo estudo de caso e controle de mortalidade infantil: Evidence for protection by breast-feeding against infant deaths from infectious diseases in Brazil em português Evidências de proteção por amamentação contra óbitos infantis de doenças infecciosas no Brasil, publicado na Lancet em 1987, citado 979 vezes.

O grupo em azul do lado esquerdo, representando a colaboração da Rede LatinoAmericana de Pesquisa Perinatal e Reprodutiva, no artigo: A Randomized Trial of Psychosocial Support during High-Risk Pregnancies em português, Um ensaio randomizado de apoio psicossocial durante gravidez de alto risco, publicado no The New England Journal of Medicine, em 1992, citado 197 vezes. E no grupo em vermelho, encontra-se a colaboração no 
artigo: Use of pacifiers and breastfeeding duration em português, Uso de chupetas e duração da amamentação, publicado na Lancet em 1993, citado 207 vezes.

Figura 3 - Infográfico da rede de colaboração de Victora, CG. 1987-1996 (Nós: autores que compõem a rede. Links: número de relações entre os autores com ligações únicas ou repetidas.)

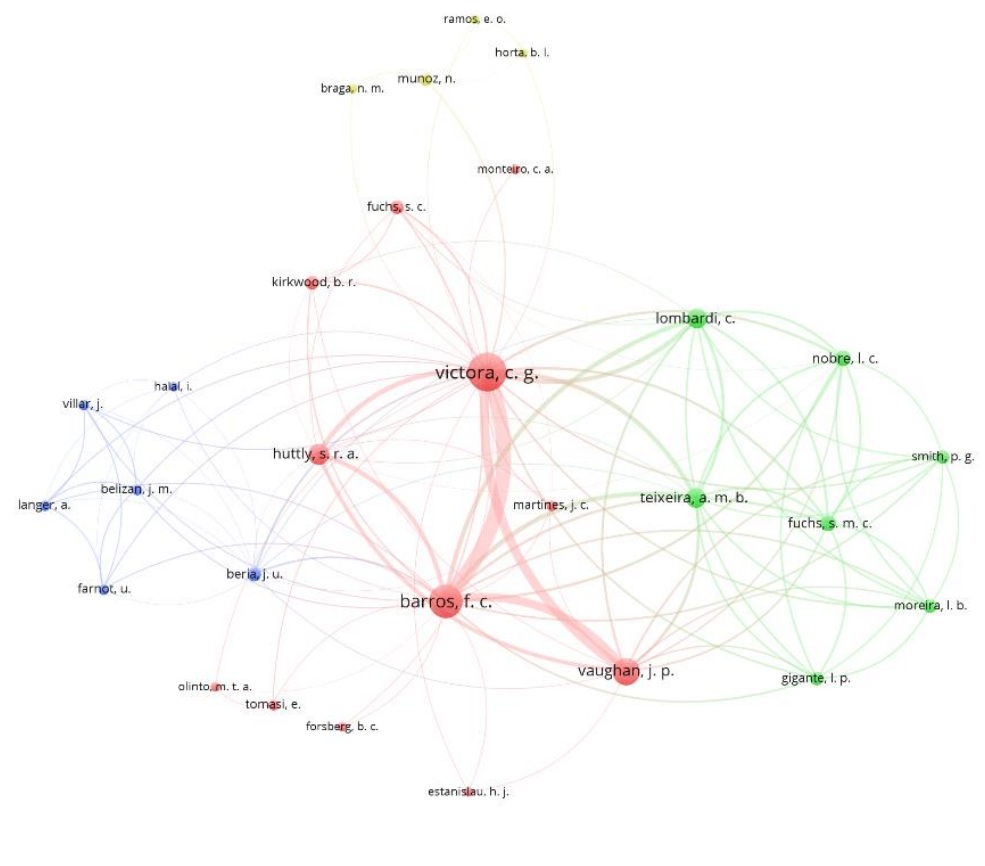

in vosviewer

Fonte de dados: elaboração própria com dados da Scopus

\subsection{A TERCEIRA DÉCADA, 1997 A 2006, A TRANSLAÇÃO DO CONHECIMENTO E A TERCEIRA COORTE DE NASCIMENTO DE PELOTAS}

Na década anterior, observamos a criação e o crescimento do Centro de Pesquisa Epidemiológicas, bem como o reflexo na produção científica, a inserção em políticas globais e impacto social.

Na terceira década, evidenciamos a consolidação do CPE como centro de excelência sendo o primeiro programa de pós-graduação a obter a nota 7 na área de Saúde Coletiva, máxima na avaliação da Capes e mantê-la até os dias atuais (CENTRO DE PESQUISA EPIDEMIOLÓGICAS UFPel, 2017). Também, ressalta-se o início da terceira coorte de nascimentos de Pelotas, neste estudo o SUS encontra-se implementado.

Na figura 4 observa-se a consolidação do CPE representado pelo grupo em vermelho. 
O grande cluster em verde, representa o grupo de trabalho: Uma curva de crescimento para o século XXI: o estudo internacional multicêntrico de crescimento infantil, consiste em um estudo epidemiológico do crescimento de crianças menores de cinco anos, a qual desenvolveu uma nova referência internacional, adotada por mais de 140 países. Victora descreve sobre este estudo em uma entrevista:

\begin{abstract}
Esse outro estudo buscava informações prescritivas de como é o crescimento ideal da espécie humana até os 5 anos. É uma curva simples: peso, idade e altura. Para estabelecer esse tipo de curva, tenho de estudar gente que cresce bem, e nas coortes tem gente que cresce bem, mal e mais ou menos. Todo mundo dizia que brasileiro é diferente de jamaicano, norueguês e indiano. Então fizemos uma coisa ambiciosa. Pegamos uma amostra de mais de 7 mil crianças do Brasil, dos Estados Unidos, da Noruega, de Omã, de Gana e da Índia. Escolhemos mães e crianças de bom nível socioeconômico - as mães tinham feito pré-natal, não eram fumantes nem tinham doenças importantes, e as crianças haviam sido amamentadas - e fomos ver como as crianças crescem. (ZORZETTO, 2013).
\end{abstract}

O cluster azul representa a rede de colaboração com Jennifer Bryce da Johns Hopkins University e outros, que resultou da Multi-Country Evaluation of IMCI (Integrated Management of Childhood Illnesses) em português Gestão Integrada de Doenças Infantis - este era um programa novo da OMS que avaliou em diversos países. No artigo The Multi-Country Evaluation of the Integrated Management of Childhood Illness Strategy: Lessons for the Evaluation of Public Health Interventions, Bryce et al. (2004) mostra a terceira vertente do trabalho de Cesar Victora ao longo do tempo: avaliação de impacto de programas de saúde. As outras duas outras vertentes são a saúde materno-infantil e as desigualdades sociais em saúde. 
Figura 4 - Infográfico da rede de colaboração de Victora, CG 1997-2006 (Nós: autores que compõem a rede. Links: número de relações entre os autores com ligações únicas ou repetidas).

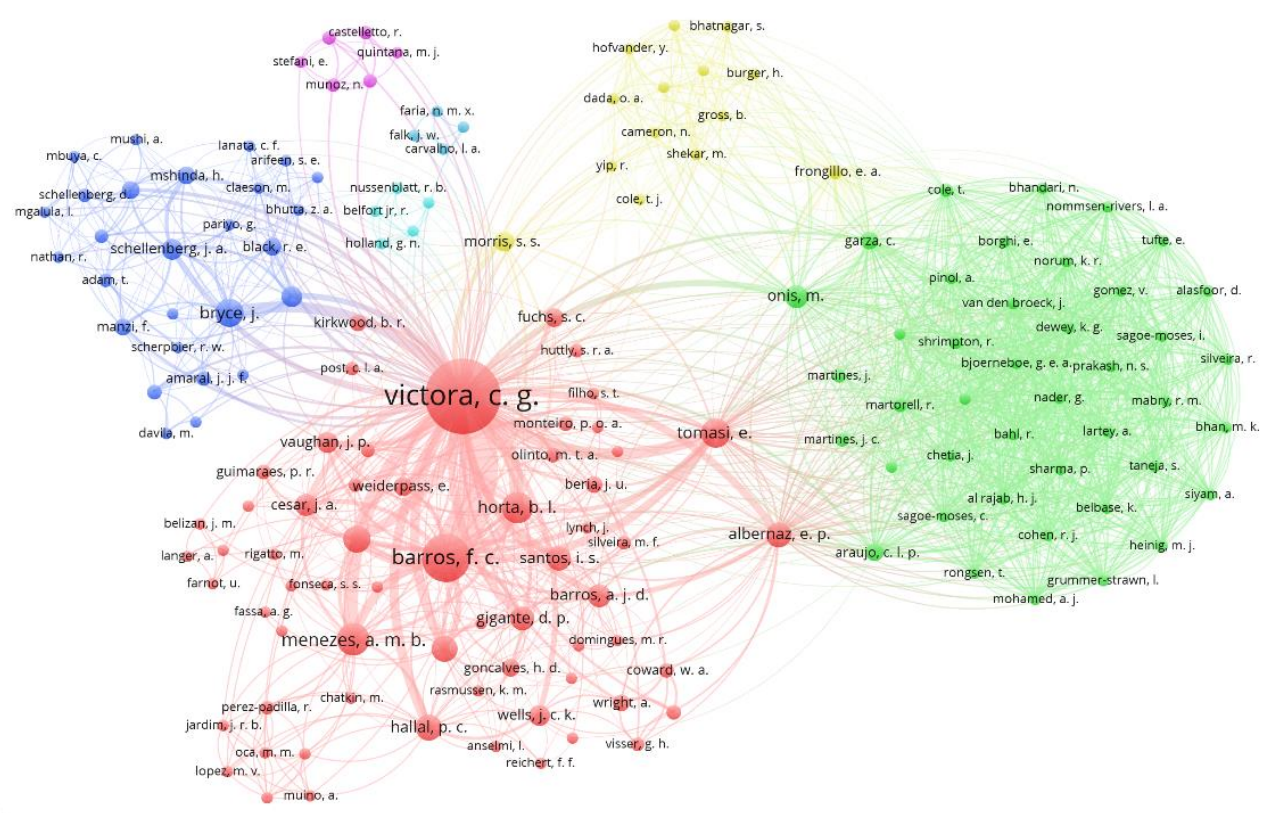

fol vosviewer

Fonte de dados: elaboração própria com dados da Scopus

\subsection{A QUARTA DÉCADA, 2007 A 2016, OS ESTUDOS MULTICÊNTRICOS E A QUARTA COORTE DE NASCIMENTO DE PELOTAS}

Nessa década, Victora amplia a rede de colaboração, aprofunda nos estudos multicêntricos, aposenta-se da UFPel em 2009, porém mantém-se ativo como coordenador do Centro Internacional de Equidade em Saúde materno-infantil e Nutrição e foi presidente da Associação Internacional de Epidemiologia de 2011 a 2014. 
Figura 5 - Infográfico da rede de colaboração de Victora, CG. 2007-2016 (Nós: autores que compõem a rede. Links: número de relações entre os autores com ligações únicas ou repetidas).

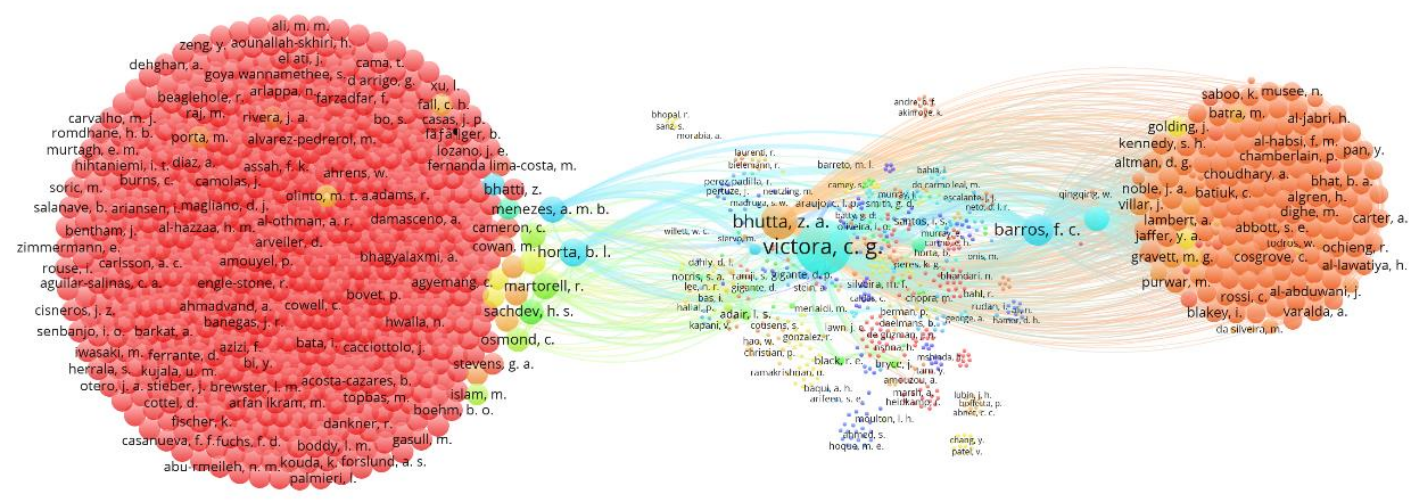

\& vosviewer

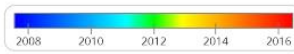

Fonte de dados: elaboração própria com dados da Scopus

Ultrasound-based gestational-age estimation in late pregnancy http://dx.doi.org/10.1002\%2Fuog.15894 A century of trends in adult human height 10.7554/eLife.13410.001

Postnatal growth standards for preterm infants: The Preterm Postnatal Follow-up Study of the intergrowth21stProject http://dx.doi.org/10.1016\%2FS2214-109X(15)00163-1

The distribution of clinical phenotypes of preterm birth syndrome implications for prevention http://dx.doi.org/10.1001\%2Fjamapediatrics.2014.3040

International standards for newborn weight, length, and head circumference by gestational age and sex: The Newborn Cross-Sectional Study of the INTERGROWTH-21st Project http://dx.doi.org/10.1016\%2Fs0140$\underline{6736(14) 60932-6}$

Na figura 6 a nova configuração apresenta o grupo em vermelho a UFPel, o grupo em azul representam as publicações sobre os Objetivos do Milênio, esta rede de colaboração evoluiu do estudo do IMCI e o ponto de inflexão foi a Série Child survival em português, Sobrevivência Infantil, publicada no Lancet em 2003, quando foi proposto a criação do Countdown to 2015, iniciativa para avaliar os objetivos do milênio. A avaliação do IMCI mostrou que muitos países não estavam conseguindo progredir adequadamente na redução da mortalidade de crianças, e o grupo evoluiu para uma iniciativa de ativismo/advocacia (o Countdown). O grupo em amarelo representa o grupo da Curva de crescimento, o grupo lilás representa o Estudo Internacional de Crescimento Fetal e do Recém-nascido Padrões para o século 21. 
Figura 6 - Infográfico da rede de colaboração de Victora, CG. 2007-2016 (Nós - autores que compõem a rede. Links - número de relações entre os autores com ligações únicas ou repetidas).

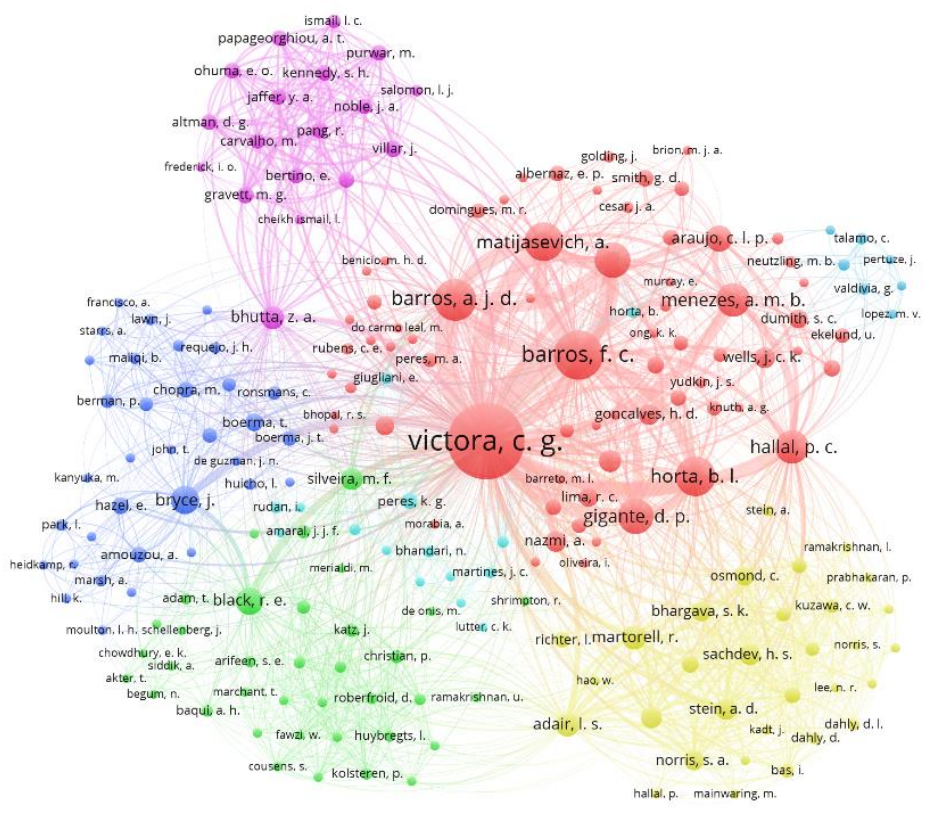

of vosviewer

Fonte de dados: elaboração própria com dados da Scopus

No quadro 1, apresenta uma síntese em formato linha do tempo, com o acúmulo do capital científico de Victora. A primeira coluna, o período de abrangência em décadas, na segunda coluna, o capital institucional, ou seja, as posições que ocupou e/ou ocupa em espaços institucionais e na terceira coluna, o capital específico ou seja, reconhecimento de pares e contribuições no avanço do conhecimento do campo que atua. Na quarta coluna, opta-se por incluir as políticas nacionais com influência para a saúde materno-infantil, para visualizar o desenvolvimento no Brasil. Entretanto algumas dessas políticas ainda que apontam diretrizes convergentes com as políticas globais, raramente citam as publicações científicas, preferindo citar documentos técnicos que também não citam publicações científicas, e assim, criam um ciclo vicioso de ausência de evidências científicas e dificultando a capacidade de monitoramento aplicabilidade dos resultados de pesquisa na sociedade. Neste sentido, sugerese para pesquisas futuras o aprofundamento no comportamento informacional do campo da saúde materno-infantil. E na quinta coluna, elencou-se as políticas globais com as quais o retratado apresenta influência. 
Quadro 1 - Linha do tempo dos principais acúmulos de capital científico de Cesar Victora e Políticas de saúde materno-infantil e contexto de outras influências

\begin{tabular}{|c|c|c|c|c|}
\hline \multirow{3}{*}{ Período } & \multicolumn{2}{|c|}{ Trajetória Cesar Gomes Victora } & \multirow{2}{*}{\multicolumn{2}{|c|}{$\begin{array}{l}\text { Políticas de Saúde materno-infantil e contexto } \\
\text { e influências }\end{array}$}} \\
\hline & \multicolumn{2}{|c|}{ Capital científico } & & \\
\hline & Institucional & Específico & Nacionais & Globais \\
\hline $\begin{array}{l}\text { Antes de } \\
1977\end{array}$ & $\begin{array}{l}\text { UFRGS - } \\
\text { Medicina } \\
(1971-1976) \\
\text { UFRGS - Saúde } \\
\text { comunitária } \\
(1976-1977)\end{array}$ & & $\begin{array}{l}\text { Expansão do } \\
\text { suprimento de água e } \\
\text { saneamento básico } \\
\text { no Brasil (1975) } \\
\text { Programa Nacional } \\
\text { de suplementação } \\
\text { alimentar para mães } \\
\text { e crianças (1976) }\end{array}$ & $\begin{array}{l}\text { Conferência de } \\
\text { Estocolmo (1972) } \\
\text { Relatório Lalonde (1974) } \\
\text { Saúde para todos } \\
(1976-2000)\end{array}$ \\
\hline $\begin{array}{c}1977 \\
a \\
1986\end{array}$ & $\begin{array}{l}\text { Professor na } \\
\text { UFPel (1977) } \\
\text { Doutorado em } \\
\text { Epidemiologia } \\
\text { LSHTM } \\
(1980-1983) \\
\text { Consultor CNPq } \\
(1983)\end{array}$ & $\begin{array}{l}\text { Início da primeira Coorte } \\
\text { de Nascimentos de } \\
\text { Pelotas com Fernando } \\
\text { Barros e J P Vaughan } \\
\text { (1982) } \\
\text { Tese: Epidemiologia da } \\
\text { saúde infantil no Rio } \\
\text { Grande do Sul, Brasil. As } \\
\text { inter-relações entre } \\
\text { mortalidade, desnutrição, } \\
\text { assistência médica e } \\
\text { desenvolvimento } \\
\text { agrícola. (1983) } \\
\text { Saúde perinatal em } \\
\text { Pelotas, RS, Brasil: } \\
\text { fatores sociais e } \\
\text { biológicos (1984) } \\
\text { Fatores de risco para a } \\
\text { desnutrição em crianças } \\
\text { brasileiras: o papel das } \\
\text { variáveis sociais e } \\
\text { ambientais - Bulletin of } \\
\text { the WHO (1986) }\end{array}$ & $\begin{array}{l}\text { Programa Nacional } \\
\text { de Imunização (1977) } \\
\text { Programa Nacional } \\
\text { de incentivo ao } \\
\text { Aleitamento Materno } \\
\text { (1981) } \\
\text { Programa Assistência } \\
\text { Integral à saúde da } \\
\text { mulher (1984) } \\
\text { Programa nacional de } \\
\text { saúde infantil (1984) }\end{array}$ & $\begin{array}{l}\text { Declaração de Alma-Ata } \\
\text { "Saúde para todos até o } \\
\text { ano } 2000(1978)\end{array}$ \\
\hline $\begin{array}{c}1987 \\
a \\
1996\end{array}$ & $\begin{array}{l}\text { Co-fundador do } \\
\text { Centro de } \\
\text { Pesquisa em } \\
\text { Epidemiologia } \\
\text { CPE UFPel (1991) } \\
\text { Coordenação do } \\
\text { programa de pós- } \\
\text { graduação em } \\
\text { epidemiologia } \\
\text { (1991-1995) } \\
\text { Consultor técnico } \\
\text { sênior da OMS } \\
\text { (1991) } \\
\text { UNICEF Consultor } \\
\text { no Brasil (1987- } \\
\text { 1991) e em Nova } \\
\text { lorque (1994) } \\
\text { LSHTM Professor } \\
\text { visitante (1996) }\end{array}$ & $\begin{array}{l}\text { Evidências de proteção } \\
\text { por amamentação contra } \\
\text { óbitos infantis de } \\
\text { doenças infecciosas no } \\
\text { Brasil - Lancet (1987) } \\
\text { Início da segunda Coorte } \\
\text { de Nascimentos de } \\
\text { Pelotas (1993) } \\
\text { Uso de chupetas e } \\
\text { duração da } \\
\text { amamentação } \\
\text { (1993) }\end{array}$ & $\begin{array}{l}\text { Programa Nacional } \\
\text { para redução da } \\
\text { mortalidade infantil } \\
\text { (1995) } \\
\text { Criação do SUS } \\
\text { (1988) } \\
\text { Criação do Programa } \\
\text { de Saúde da Família } \\
\text { (1994) }\end{array}$ & $\begin{array}{l}\text { Alimentação infantil: a } \\
\text { base fisiológica (1990) } \\
\text { Physical status: The use } \\
\text { and interpretation of } \\
\text { anthropometry WHO } \\
\text { (1995) }\end{array}$ \\
\hline
\end{tabular}




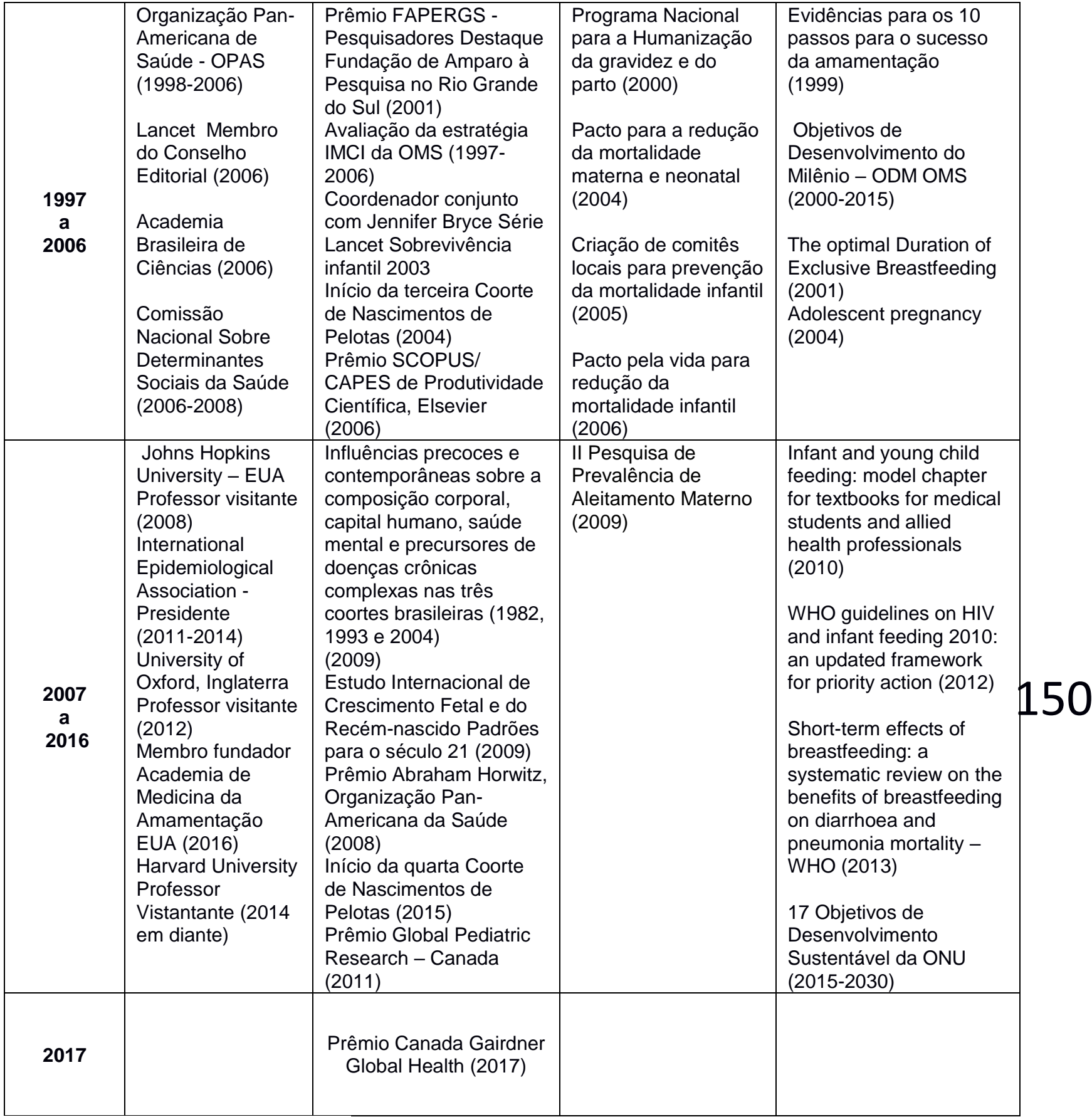

Fonte de dados: elaboração própria

\subsection{LIMITAÇÕES DO ESTUDO}

As principais limitações deste estudo são: 1) a qualidade dos dados dos nomes dos autores. Apesar de ter sido realizada a limpeza e padronização dos nomes, considera-se a possibilidade de ter ambiguidade nos nomes dos autores nas referências recuperadas. 2) As redes de co-autoria não expressam a totalidade da produção científica de Cesar Gomes Victora, não foram incluídos os livros, os documentos técnicos e as orientações acadêmicas. 3) A 
colaboração científica não está limitada a autoria, representa um aspecto da colaboração científica.

\section{CONCLUSÃO}

Este estudo buscou identificar o capital científico e a evolução da rede de colaboração científica de uma autoridade do campo da Saúde Materno infantil ao longo de 40 anos - Cesar Victora, por meio da análise bio-bibliométrica. Observou-se o crescimento exponencial do número de publicações, bem como de colaborações. Ressalta-se a força da ligação com o local de origem, UFPel durante os 40 anos e as demais instituições internacionais como a Organização Mundial de Saúde e LSHTM. Observa-se que o retratado manteve em forte ligação com a agenda de pesquisa internacional desde o início da carreira sendo fortalecida ao longo dos quarenta anos. $\mathrm{O}$ acúmulo do capital científico tanto institucional, como específico foram construídos em contextos local e global conferindo ao Cesar Gomes Victora autoridade internacional no campo materno-infantil.

As análises bio-bibliométricas de autoridades de campos de conhecimento possibilitam compreender o movimento das colaborações e do acumulo de capital científico. Este método pode ser utilizado para apoiar a visualização da informação em memoriais acadêmicos, bem como incrementar artigos históricos com finalidade de retratar um personagem. 


\section{REFERENCIAS}

BARROS, Fernando C. et al. Saúde perinatal em Pelotas, RS, Brasil: fatores sociais e biológicos. Revista de Saúde Pública, [s.1.], v. 18, n. 4, p.301-312, ago. 1984. FapUNIFESP (SciELO). http://dx.doi.org/10.1590/s0034-89101984000400005

BOSELEY, Sarah. Cesar Victora: leader in child health and development. The Lancet, [s.1.], v. 369, n. 9555, p.21-21, jan. 2007. Elsevier BV. http://dx.doi.org/10.1016/s01406736(07)60015-4.

BOURDIEU, Pierre. O campo científico. In. ORTIZ, Renato. (Org.). Pierre Bourdieu: sociologia. São Paulo: Ática, 1983. Cap. 4, p. 122-155

BOURDIEU, Pierre. Os Usos sociais da ciência: por uma sociologia clínica do campo científico. São Paulo: Unesp; 2004.

BRYCE, Jennifer et al. "The Multi-Country Evaluation of the Integrated Management of Childhood Illness Strategy: Lessons for the Evaluation of Public Health

Interventions.” American Journal of Public Health 94.3 (2004): 406-415. Disponível https://www.ncbi.nlm.nih.gov/pmc/articles/PMC1448266/

CANADÁ. New Perspective on the Health of Canadians: a working document. Ottawa. 1974. Disponível: http://www.phac-aspc.gc.ca/ph-sp/pdf/perspect-eng.pdf

EPIDEMIOLOGIA UFPel http://epidemio-ufpel.org.br/site/content/cpe/index.php

GONZÁLEZ-ALCAIDE, Gregorio et al. Evolution of Cooperation Patterns in Psoriasis Research: Co-Authorship Network Analysis of Papers in Medline (1942-2013). Plos One, [s.l.], v. 10, n. 12, p.1-19, 11 dez. 2015. Public Library of Science (PLoS). http://dx.doi.org/10.1371/journal.pone.0144837.

HAYASHI, Maria Cristina Piumbato Innocentini. Fertilizações cruzadas entre a cientometria, a sociologia da ciência e os estudos sociais da ciência. In: HAYASHI, Maria Cristina Piumbato Innocentini; RIGOLIN, Camila Carneiro Dias; KERBAUY, Maria Teresa Miceli (Org.). Sociologia da Ciência: contribuições ao campo CTS. Campinas: Alínea, 2014. p.267-305.

KLOTZEL, Daniel. Homenagem a Kurt Kloetzel (1923-2007). Interface - Comunicação, Saúde, Educação, [s.1.], v. 11, n. 23, p.673-677, dez. 2007. FapUNIFESP (SciELO). http://dx.doi.org/10.1590/s1414-32832007000300025.

MINISTÉRIO DA SAÚDE. Assistência integral à saúde da criança: ações básicas/ Ministério da Saúde. Brasília, Centro de Documentação do Ministério da Saúde, 1984. Série B: Textos Básicos de Saúde, 7. Disponível:

http://bvsms.saude.gov.br/bvs/publicacoes/assistencia_integral_saude_crianca.pdf

MOED, Henk F., GLÄNZEL, Wolfgang, SCHMOCH, Ulrich (Org.). Handbook of quantitative science and technology research: the use of publication and patent statistics in studies of S\&T systems. Dordrecht: Kluwer Acad. Publ; 2004. 800 p. 
Series Lancet Child survival 2003 Disponível https://www.thelancet.com/series/childsurvival

VICTORA Cesar Gomes et al. Estudo longitudinal das crianças nascidas em 1982 em Pelotas, RS, Brasil: metodologia e resultados preliminares. Rev. Saúde Pública [Internet]. 1985 Feb [cited 2017 July 24] ;19( 1 ): 58-68. http://dx.doi.org/10.1590/S0034-89101985000100007.

VICTORA, Cesar Gomes et al. Risk factors for malnutrition in Brazilian children: the role of social and environmental variables. Bulletin of the World Health Organization. 1986;64(2):299-309.

VICTORA, Cesar Gomes et al. Evidence for protection by breast-feeding against infant deaths from infectious diseases in Brazil. Lancet (British edition), v. 330, p. 319-322, 1987.

VILLAR, José et al. A Randomized Trial of Psychosocial Support during High-Risk Pregnancies. New England Journal Of Medicine, [s.1.], v. 327, n. 18, p.1266-1271, 29 out. 1992. http://dx.doi.org/10.1056/nejm199210293271803.

ZORZETTO Ricardo. Cesar Victora: A saúde por trás das estatísticas. Revista Pesquisa Fapesp. 2013 Disponível http://revistapesquisa.fapesp.br/2013/06/05/cesar-victora-a-saudepor-tras-das-estatisticas/ 